Journal Club

Editor's Note: These short, critical reviews of recent papers in the Journal, written exclusively by graduate students or postdoctoral fellows, are intended to summarize the important findings of the paper and provide additional insight and commentary. For more information on the format and purpose of the Journal Club, please see http://www.jneurosci.org/misc/ifa_features.shtml.

\title{
Prelimbic Prefrontal Neurons Drive Fear Expression: A Clue for Extinction-Reconsolidation Interactions
}

\author{
Daniela Schiller ${ }^{1,2}$ and Joshua Johansen ${ }^{1}$ \\ ${ }^{1}$ Center for Neural Science and ${ }^{2}$ Department of Psychology, New York University, New York, New York 10003 \\ Review of Burgos-Robles et al.
}

Learning associations between cues in the environment gives organisms the ability to predict impending danger. The last few decades of research have established that these stimulus-danger or fear associations are formed in the amygdala (LeDoux, 2000). This type of learning is modeled in the laboratory by presenting a neutral sensory stimulus (such as a tone) with an aversive stimulus (such as a shock) in close temporal contiguity. As a result, organisms learn to fear the previously neutral stimulus. The convergence of afferent inputs signaling the neutral stimulus (termed the conditioned stimulus or CS) and the aversive stimulus (unconditioned stimulus or US) onto neurons in the lateral amygdala (LA) leads to the long-term potentiation (LTP) of CS input synapses, such that when the CS subsequently occurs on its own, these inputs are now strong enough to drive outputs from the LA to trigger the fear response. The fear response is mediated through the major output structure of the amygdala, the central nucleus, and comprises a wide repertoire of behavioral and physiological reactions including behavioral freezing, changes in heart rate and blood pressure, and release of stress hormones. But whereas CS-evoked fear reactions, such as freezing, are long lasting

Received Aug. 31, 2009; revised Sept. 22, 2009; accepted Sept. 28, 2009. Correspondence should be addressed to Daniela Schiller, Center for Neural Science, New York University, 4 Washington Place, Room 809, New York, NY, 10003. E-mail: schiller@cns.nyu.edu.

DOI:10.1523/JNEUROSCI.4299-09.2009

Copyright $\odot 2009$ Society for Neuroscience $\quad$ 0270-6474/09/2913432-03\$15.00/0 and slow to dissipate (lasting at least as long as the CS, that is, a few seconds to several minutes, and in some cases longer), LA neural responses during the CS are brief and phasic ( 50-500 ms) (Quirk et al., 1995). Thus, while the association of the CS and US are formed in the LA (and possibly elsewhere) and neural output from the LA is required to initiate the fear response, the expression and maintenance of the fear response to the CS is sustained somewhere else. Though individual brain regions have been identified that participate as output structures for specific reactions to fearful stimuli (such as the midbrain periaqueductal gray for conditioned freezing and analgesia), the specific mechanisms by which these fearconditioned responses are maintained are not well understood. This has prompted recent studies examining possible brain regions that function to sustain the fear response throughout the $\mathrm{CS}$, and has led to the discovery of the prelimbic cortex (PL) as a likely candidate for this role. Previous studies showed that inactivation of PL reduced conditioned fear responses, whereas stimulation of PL enhanced fear responses (Corcoran and Quirk, 2007; Vidal-Gonzalez et al., 2006).

Additional evidence that directly linked PL activation and sustained conditioned fear was reported recently in The Journal of Neuroscience by Burgos-Robles et al. (2009). Using multichannel unit recordings in behaving rats, the authors characterized the firing profile of PL neurons throughout fear conditioning and ex- tinction. They reported three key findings: (1) PL neurons showed conditioninginduced enhancement of neural firing rate in response to the CS that diminished with extinction; (2) These conditioned responses were sustained throughout the presentation of the conditioned stimuli (a $30 \mathrm{~s}$ tone) and correlated with the expression of CS-induced freezing behavior; (3) The magnitude of CS-induced PL responses during fear conditioning corresponded with the ability to successfully recall extinction memory $24 \mathrm{~h}$ later. Together, these findings provide striking support for the idea that PL neurons drive the expression of conditioned fear.

The PL is well situated for this role because it receives inputs from the hippocampus and sensory cortex, and it is the target of neuromodulators such as norepinephrine and dopamine that are released in response to frightening stimuli and might participate in the maintenance of the fear response. PL also sends outputs to the basal nucleus of the amygdala, as well as to other regions mediating not only conditioned freezing (periaqueductal gray) but also conditioned instrumental responses (ventral striatum) such as active avoidance. Based on this architecture, Burgos-Robles et al. (2009) proposed a model (their Fig. 7) wherein PL converts phasic inputs from the amygdala into sustained outputs.

The transition to nonfearful memories To function efficiently in a changing environment, animals must not only be able to 
express fear, but also know when not to express it. This knowledge is hypothesized to be acquired through extinction learning. During extinction training, an animal learns that the sensory event that previously predicted impending danger is no longer associated with these aversive outcomes. Through a variety of approaches, it has become clear that extinction learning is not simply an unlearning of the previously learned associations (i.e., a depotentiation of the originally potentiated synapses), but rather the formation of a new memory that serves to inhibit the original fear memory. A wealth of studies have revealed that the infralimbic cortex (IL), which is directly adjacent to the PL, is intimately involved in the consolidation of extinction and that plasticity in the amygdala (possibly in inhibitory interneurons) is also required for this process (Lin et al., 2003; Paré et al., 2004; Myers and Davis, 2007; Quirk and Mueller, 2008).

In addition to showing that PL neurons are involved in the expression of conditioned fear, Burgos-Robles et al. (2009) suggest that PL and IL are involved in regulating the balance between fearful and nonfearful memories. Their data indicate a negative correlation between PL activity and extinction success. Specifically, strong PL activation by the CS was related to failure to express extinction not only when tested a day after training but also during the training session. Based on these findings, the authors speculated that extinction failure might result not only from reduced activity in IL, but also from an excessively active PL, and perhaps more critically, on the balance between these two neighboring regions. Yet, how these effects come about and the role that PL plays in extinction is currently unclear.

A different mechanism for maintaining the balance between fear and nonfear states was suggested by Herry et al. (2008), who found that separate populations of neurons in the basal amygdala trigger transitions between high and low fear states. One set of neurons exhibited enhanced CS-evoked responses after fear conditioning and renewal and the other population showed enhanced CS-evoked activity after fear extinction. These authors also found that temporary inactivation of basal amygdala neurons blocked the acquisition of fear extinction learning as well as renewal of extinguished fear, suggesting that the two different types of neurons are involved in behavioral transitions between high and low fear states. Together, these brain regions (the PL and basal amygdaloid nucleus) may participate in regulating the balance between high and low fear states and the expression of fear and extinction memories.

\section{Changing fear memories through reconsolidation}

The relation between fear expression and extinction is highly relevant to an independent set of findings involving memory reconsolidation. During reconsolidation, stored information is rendered labile after being retrieved. Pharmacological perturbations at this stage result in an inability to retrieve the memories at later times, suggesting they are either persistently inhibited or even erased (Nader et al., 2000). It has been suggested that the adaptive function of reconsolidation is to allow new information available at the time of retrieval to be integrated into the memory representation (Dudai, 2006).

Monfils et al. (2009) have attempted to make constructive use of the vulnerability window during reconsolidation to block the expression of fear memories. In brief, $24 \mathrm{~h}$ after fear conditioning, rats were reminded of the CS using a single unreinforced presentation, and subsequently underwent extinction training. The reconsolidation window lasts $\sim 6 \mathrm{~h}$, and so the extinction phase was conducted either within or outside of this window. Rats that had extinction training during the reconsolidation window did not show recovery of fear. Only rats that underwent extinction after reconsolidation was completed showed a return of fear responses. In addition, rats that underwent extinction within the reconsolidation window exhibited retarded relearning when conditioned again with the same CS compared with animals that underwent extinction outside the reconsolidation window or those undergoing fear conditioning for the first time. The authors hypothesized that the information learned during extinction training was integrated into the recalled fear memory through reconsolidation, resulting in alteration of the original fear memory into a nonfearful form. In contrast, they concluded that extinction training that occurred outside the reconsolidation phase produced normal extinction learning as described above. In the latter process, the CS-US association remained intact and could therefore resurface under certain conditions, such as exposure to stress (reinstatement) or mere passage of time (spontaneous recovery). According to these results, to prevent the recovery of extinguished fear, extinction training must be preceded by a reac- tivation of the fear memory. The reason the first stimulus in extinction training does not produce the same result might have to do with the molecular mechanism triggered by the different timing schemes: Monfils at al. (2009) found that memory retrieval resulted in increased synaptic plasticity in the LA (as indexed by phosphorylation of the AMPA receptor subunit GluR1), and that this plasticity was reversed by the presentation of another CS an hour later but not by a CS presented 3 min later, as occurs during extinction training.

This study suggests the intriguing hypothesis that when extinction training occurs during reconsolidation, the neural processes that normally mediate extinction learning are subjugated, and instead mechanisms are engaged which serve to reconsolidate the original memory into a nonfearful form. The observation by Burgos-Robles et al. (2009) that rats with higher PL responses during extinction training later failed to recall extinction suggests that PL (and possibly basal amygdala) signaling during retrieval might play a role in disabling the extinction learning circuit when extinction training occurs during reconsolidation. One prediction of this hypothesis is that the magnitude of $\mathrm{PL}$ signaling during retrieval might be increased and correlate with the blockade of fear responses when extinction training is conducted during reconsolidation. While these circuits could inhibit normal extinction learning, another mechanism may reform the original fear memory. This could be accomplished through depotentiation or long-term depression of the CS input synapses on LA neurons that were originally strengthened during fear memory formation. In fact, there is evidence that under certain conditions, extinction can trigger depotentiation at LA synapses (Kim et al., 2007). It is possible that depotentiation is enhanced when extinction training occurs during reconsolidation. Memory retrieval engages both reconsolidation and extinction processes but perhaps the delay between retrieval and the subsequent extinction training enables a dormant or a nondominant depotentiation process to occur.

In conclusion, these recent studies have begun to elucidate the neural mechanisms mediating the transition and relationship between fearful and nonfearful memories. Findings about the role of PL in sustaining conditioned fear, the IL in recall of extinction, and the amygdala in the acquisition of both begin to portray the particular balance required to express 
each state. Further examination of how these systems interact to produce integration of fear and nonfear memories will be important work for future studies.

\section{References}

Burgos-Robles A, Vidal-Gonzalez I, Quirk GJ (2009) Sustained conditioned responses in prelimbic prefrontal neurons are correlated with fear expression and extinction failure. J Neurosci 29:8474-8482.

Corcoran KA, Quirk GJ (2007) Activity in prelimbic cortex is necessary for the expression of learned, but not innate, fears. J Neurosci $27: 840-844$.

Dudai Y (2006) Reconsolidation: the advantage of being refocused. Curr Opin Neurobiol 16:174-178.

Herry C, Ciocchi S, Senn V, Demmou L, Müller C, Lüthi A (2008) Switching on and off fear by distinct neuronal circuits. Nature 454:600606.

Kim J, Lee S, Park K, Hong I, Song B, Son G, Park H, Kim WR, Park E, Choe HK, Kim H, Lee C, Sun W, Kim K, Shin KS, Choi S (2007) Amygdala depotentiation and fear extinction. Proc Natl Acad Sci U S A 104:20955-20960.

LeDoux JE (2000) Emotion circuits in the brain. Annu Rev Neurosci 23:155-184.

Lin CH, Yeh SH, Lu HY, Gean PW (2003) The similarities and diversities of signal pathways leading to consolidation of conditioning and consolidation of extinction of fear memory. J Neurosci 23:8310-8317.

Monfils MH, Cowansage KK, Klann E, LeDoux JE (2009) Extinction-reconsolidation boundaries: key to persistent attenuation of fear memories. Science 324:951-955.

Myers KM, Davis M (2007) Mechanisms of fear extinction. Mol Psychiatry 12:120-150.
Nader K, Schafe GE, Le Doux JE (2000) Fear memories require protein synthesis in the amygdala for reconsolidation after retrieval. Nature 406:722-726.

Paré D, Quirk GJ, Ledoux JE (2004) New vistas on amygdala networks in conditioned fear. J Neurophysiol 92:1-9.

Quirk GJ, Mueller D (2008) Neural mechanisms of extinction learning and retrieval. Neuropsychopharmacology 33:56-72.

Quirk GJ, Repa C, LeDoux JE (1995) Fear conditioning enhances short latency auditory responses of lateral amygdala neurons: parallel recordings in the freely behaving rat. Neuron 15:1029-1039.

Vidal-Gonzalez I, Vidal-Gonzalez B, Rauch SL, Quirk GJ (2006) Microstimulation reveals opposing influences of prelimbic and infralimbic cortex on the expression of conditioned fear. Learn Mem 13:728-733. 